\title{
Speed of information processing as a key deficit in multiple sclerosis: implications for rehabilitation
}

\author{
Heath A Demaree, John DeLuca, Elizabeth A Gaudino, Bruce J Diamond
}

\begin{abstract}
Speed of information processing was assessed in patients with multiple sclerosis and healthy controls using both an auditory and visual task designed to control for accuracy of performance across groups. After controlling for accuracy of performance, patients with multiple sclerosis were found to have significantly slower speed of information processing relative healthy controls, irrespective of the modality of stimulus presentation (auditory or visual). When given an adequate amount of time to process information, however, the patients performed similarly to controls. These results suggest that persons with multiple sclerosis experience deficits specifically in processing speed but not performance accuracy. Results are discussed in terms of rehabilitative guidelines for the cognitive improvement of persons with multiple sclerosis.

(F Neurol Neurosurg Psychiatry 1999;67:661-663)
\end{abstract}

Kessler Medical

Rehabilitation

Research and

Education

Corporation,

Neuropsychology and

Neuroscience

Laboratory, West

Orange, NJ, USA, and

UMDNJ-New Jersey

Medical School,

Departments of

Physical Medicine and

Rehabilitation, and

Neurosciences,

Newark, NJ, USA

$\mathrm{H}$ A Demaree

J DeLuca

E A Gaudino

B J Diamond

Correspondence to: Dr John DeLuca,

Neuropsychology Labratory,

Kessler Medical

Rehabilitation Research and

Education Corporation,

1199 Pleasant Valley Way,

West Orange, New Jersey

07052. E-mail:

delucajo@umdnj.edu

Received 27 November 1998 and in final form

26 April 1999

Accepted 6 May 1999
Keywords: multiple sclerosis; speed of information processing; rehabilitation; working memory; learning

Prior researchers, using different tests, have suggested that decreased speed of information processing is a primary deficit in multiple sclerosis. $^{1-5}$ For example, using the Sternberg memory scanning test, Rao et al found that patients with multiple sclerosis required more time than control subjects to determine whether a specific numeral was included in a number set that they were asked to remember. ${ }^{6}$ Because the two groups had similar accuracy levels, however, the authors suggested that persons with multiple sclerosis may primarily have information processing deficits. Although these results provided important information, it should be noted that the methods used in this as well as most other studies have what is known as the "speed versus accuracy confound". Also these studies are unable to quantify information processing speed while controlling for accuracy.

Attention and complex information processing have been assessed using various neuropsychological measures, including the digit span portion of the Wechsler adult intelligence scale (WAIS), the trail making test, the Stroop colour word interference test, the Sternberg memory scanning test, and the paced auditory serial addition test (PASAT). Both speed and accuracy of performance on such measures have been used as indicators of slower processing speed among patients with multiple sclerosis relative to healthy controls.

Because it is not confounded by visuomotor abilities, the PASAT is a particularly attractive method for assessing cognitive processing speed in multiple sclerosis. However, as in the other tasks purported to measure information processing speed, the traditional PASAT has a trade off between speed and accuracy of performance. That is, faster presentation rates are associated with decreased accuracy of performance. Measurement of speed of information processing while controlling for accuracy is therefore impossible using the standard PASAT protocol.

The present study was designed to address the need for an assessment of speed of information processing which is not confounded by accuracy. Specifically, a PASATlike protocol is used which is designed to measure speed of information processing by adjusting the presentation rate to control for accuracy of performance between patients with multiple sclerosis and control subjects. ${ }^{14}$ Relative to healthy controls, patients with multiple sclerosis were hypothesised to show a slower rate of processing when accuracy was equated between the two groups. However, when using a person's optimum interstimulus interval, it was predicted that patients with multiple sclerosis would perform as accurately as healthy controls.

\section{Methods}

PARTICIPANTS

Procedures were approved by the Institutional Review Board of the University of Medicine and Dentistry of New Jersey-New Jersey Medical School and Kessler Medical Rehabilitation Research and Education Corporation. Eighty one patients with clinically definite multiple sclerosis and 36 healthy control subjects comprised the sample. ${ }^{8}$ No significant group differences were found with regard to any demographic variable, including a measure of visuospatial perception (judgement of line orientation test; JLO) and an overall cognitive screening measure (cognitive capacity screen- 
Table 1 Demographic data for multiple sclerosis (MS) and healthy control (HC) groups

\begin{tabular}{lll}
\hline & $\begin{array}{l}\text { MS }(n=81) \\
\text { Mean (SEM) }\end{array}$ & $\begin{array}{l}\text { HC }(n=36) \\
\text { Mean }(\text { SEM })\end{array}$ \\
\hline Age & $45.57(10.8)$ & $42.64(11.3)$ \\
Education & $14.99(2.2)$ & $15.00(2.4)$ \\
CCSE & $27.79(.22)$ & $28.00(0.30)$ \\
JLO & $22.98(0.48)$ & $22.72(0.76)$ \\
EDSS & $5.3(1.4)$ & - \\
Years since diagnosis & $7.3(7.3)$ & - \\
Women (\%) & 77 & 81 \\
Right handed (\%) & 95 & 97 \\
\hline
\end{tabular}

ing examination; CCSE). Demographic data are presented in the table.

The multiple sclerosis participants, who were at least 1 month after exacerbation or use of corticosteroids, consisted of 32 relapsingremitting, 20 primary progressive, and $29 \mathrm{sec}-$ ondary progressive subtypes. Healthy controls were either hospital employees or people in the community recruited via newspaper advertisement, and were screened to exclude those with a neurological or psychiatric history. Multiple sclerosis and healthy control groups had no history of alcohol or drug misuse, psychiatric disorders, or loss of consciousness. All subjects gave their Internal Review Board approved informed consent before the beginning of the experiment.

To compare groups on both the auditory and visual domains, patients with multiple sclerosis and healthy controls were divided into two groups - one group was administered the auditory threshold serial addition test (AT-SAT) ( $\mathrm{n}=55$ patients with multiple sclerosis and 16 healthy control subjects) and the other group was administered the visual threshold serial addition test (VT-SAT) $(\mathrm{n}=26$ patients with multiple sclerosis and 20 healthy control subjects). Because we had no prior information regarding the mean and variance expected from the AT-SAT and VT-SAT data, a power analysis was not carried out before testing and was not responsible for the discrepant cell sizes in this research. Rather, the combination of two different investigative research protocols-one designed to measure speed of information processing in the visual domain and the other in the auditory domain-resulted in discrepant cell sizes. The two multiple sclerosis groups did not differ on mean expanded disability status scale (EDSS) scores, ${ }^{9}$ CCSE, duration of illness, or disease course.

\section{MATERIALS AND PROCEDURE}

Subjects were administered one of two computerised tasks - the AT-SAT or the VT-SAT. These programs were created to allow for the assessment of speed of processing while controlling for accuracy of performance. ${ }^{10}$ Administration procedures for the AT-SAT and VT-SAT portions of this procedure were similar to the standardised, published protocol of the PASAT. ${ }^{11}$ As in the PASAT, subjects were instructed to add each presented number (ranging from 1 to 9 ) to the number immediately preceding it, and to say the sum aloud, repeating the operation consecutively. On the VT-SAT, presented numbers measured $5 \mathrm{~mm}$ in height and were presented centrally on a computer monitor. On AT-SAT and VT-SAT trials, 50 numbers were sequentially presented which required 49 responses from each subject.

Both the AT-SAT and the VT-SAT utilise two trials of presentation. Trial 1 within each modality used a method of limits procedure to determine the rate of stimulus presentation for each subject to achieve a $50 \%$ success rate. This interstimulus interval, referred to as threshold speed (or "optimal" speed), represents an index of speed of information processing while controlling for accuracy of performance. The second trial was administered to examine the hypothesis that the multiple sclerosis group would evidence statistically similar accuracy rates relative to the human control group when each subject's predetermined individual threshold speed obtained from trial 1 was used as the presentation speed. Thus in trial 2, all subjects were evaluated to determine their percentage of correct responses, known as accuracy of performance, at their individual optimal threshold speeds.

\section{Results}

As expected, a two way (group and modality) analysis of variance (ANOVA) showed a significant main effect for group $(F(1$, $115)=6.22, p=0.014)$ with the multiple sclerosis group ( $M=2405.5$ (SEM 14.3) ms) having a significantly longer (slower) interstimulus interval relative to the healthy control group ( $M=1994.8$ (SEM 13.0) ms; figure). A significant main effect of modality $(F(1,115)=38.20$, $\mathrm{p}<0.001$ ) was also found (figure). Specifically, slower threshold speeds were obtained for the AT-SAT ( $M=2709.0$ (SEM 14.2) ms), in comparison with the VT-SAT $(M=1691.3$ (SEM 13.1) $\mathrm{ms}$ ).

The interaction effect between group and modality on threshold speed was not significant $(F(1,113)=0.73, \mathrm{p}=0.393)$.

A two way (group and modality) ANOVA examining accuracy of performance during trial 2 showed no significant main effect of group $(F(1,115)=0.61, \mathrm{p}=0.436)$ or interaction effect between group and modality $(F(1,113)=0.15, \mathrm{p}=0.702)$. Thus, when all subjects were allowed to perform the serial addition tests at their own optimal threshold speed, multiple sclerosis and healthy control groups were statistically indistinguishable. However, a main effect of modality was found

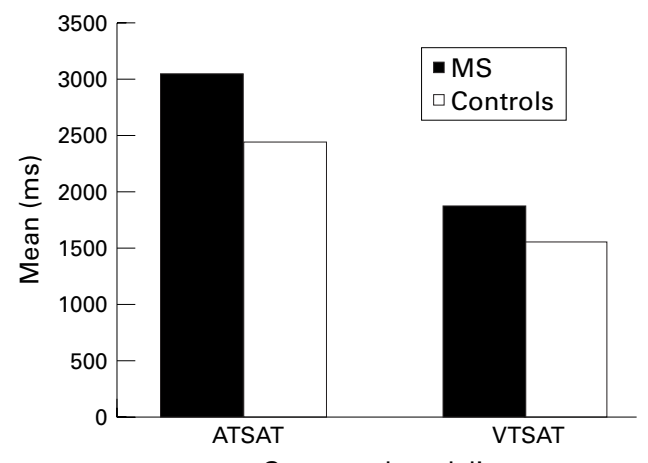

Group and modality

Mean threshold speed for patients with multiple sclerosis and healthy control subjects on the AT-SAT and VT-SAT. 
$(F(1,115)=4.74, \mathrm{p}=0.032)$, with a greater percentage of correct trials achieved for the VT-SAT $(M=51.0$ (SEM 0.008)\%) relative to the AT-SAT $(M=48.5$ (SEM 0.010)\%). Although this effect was statistically significant, the difference between an accuracy rate of $48.5 \%$ and $51.0 \%$ has no substantive clinical implications.

\section{Discussion}

Because of the protocol's ability to assess processing speed while controlling for accuracy, the present results suggest that working memory deficits in multiple sclerosis were due to impaired speed, and not to accuracy of performance. The results of the present study correspond well with prior research indicating that patients with multiple sclerosis experience decreased speed of information processing relative to healthy controls. ${ }^{312}$

Trial 2 data are remarkable for showing that the multiple sclerosis group performs as accurately as the healthy control group on both the AT-SAT and VT-SAT when they are allowed to use more time to process information (use of their individual optimal threshold speed). That is, when working memory accuracy was controlled, it was determined that speed of information processing was the primary problem in persons with multiple sclerosis.

The results of the present study show that performance accuracy on cognitive tasks can improve significantly when patients with multiple sclerosis are provided additional time to process information. One reason why this may improve performance is that the additional time strengthens the encoding of information in working memory. For instance, whereas it is well established that patients with multiple sclerosis perform poorly on tests of information recall, ${ }^{13}$ recent data suggest that the problem is in the acquisition (or learning) of information and not retrieval from long term storage. ${ }^{214}$ That is, when patients with multiple sclerosis are provided additional acquisition trials to equate level of learning with healthy controls, recall performance in multiple sclerosis does not differ from that found in controls. DeLuca et al suggest that the additional acquisition trials "provide the opportunity to improve the quality of encoding of the to-be-learned information". ${ }^{14}$ The results of the present study suggest that speed of information processing is a key factor which influences encoding in working memory.

The present data provide the groundwork for a cognitive rehabilitation programme based on maximising encoding by providing subjects more time to process information. The few prior attempts to identify the important factors in the cognitive rehabilitation of patients with multiple sclerosis have been relatively unsuccessful. Jonsson et al however, found that the only patients with multiple sclerosis to improve with regard to visuospatial memory were those who were "urged to work slowly and systematically". ${ }^{15}$ This may suggest that improved visuospatial memory performance may have resulted from decreased time pressure on working memory systems. Taken together, the results from the present study on speed of processing coupled with prior research indicating that additional stimulus exposure improves encoding and recall suggest that patients with multiple sclerosis could benefit significantly from a cognitive rehabilitation programme that improves the encoding of to be learned information.

This work was funded in part by grant No RG 2596A1/3 from the National Multiple Sclerosis Society and a UMDNJ Foundation grant No 411-68-0527. This paper was presented cal Society, Washington DC, USA, May 1998.

1 Rao SM, Leo GJ, Bernardin L, et al. Cognitive dysfunction in multiple sclerosis. I. Frequency, patterns, and prediction. Neurology 1991;41:685-91.

2 DeLuca J, Barbieri-Berger S, Johnson SK. The nature of memory impairments in multiple sclerosis: acquisition versus retrieval. F Clin Exp Neuropsychol 1994;18:56-62.

3 Diamond BJ, DeLuca J, Kim H, et al. The question of disproportionate impairments in visual and auditory processng in patients with multiple sclerosis. F Clin Exp Neuropsychol 1997;19:34-42.

4 Troyer AK, Fisk JD, Archibald CJ, et al. Conceptual reasoning as a mediator of verbal recall in patients with multiple sclerosis. Arch Neurol 1996;49:641-3.

5 Kujala P, Portin R, Revonsuo A, et al. Attention related performance in two cognitively different subgroups or patients with multiple sclerosis. $\mathcal{F}$ Neurol Neurosurg Psychiatry 1995; 59:77-82.

6 Rao SM, St Aubin-Faubert P, Leo GJ. Information processing speed in patients with multiple sclerosis. $f$ Clin Exp Neuropsychol 1989;11:471-7.

7 Madigan NK, DeLuca J, Diamond BJ, et al. Speed of information processing in traumatic brain injury: a modality specific impairment.

8 Poser CM, Paty DW, Sheinberg L. New diagnostic criteria for multiple sclerosis: guidelines for research protocols. Ann Neurol 1983:13:227-31.

9 Kurtzke JF. Rating neurologic impairment in multiple Kurtzke JF. Rating neurologic impairment in multiple
sclerosis: an expanded disability status scale (EDSS). Neusclerosis: an expanded disabil
rology 1983;33:1444-542.

10 Diamond BJ, DeLuca J, Johnson SK, et al. Processing speed, memory and executive function in Huntington's disease. fournal of the International Neuropsychological Society 1998;4:64-5.

1 Brittain JL, LaMarche JA, Reeder KP, et al. Effects of age and IQ on paced auditory serial addition task (PASAT) performance. The Clinical Neuropsychologist 1991;5:16375.

12 DeLuca J, Johnson SK, Natelson BH. Information processing efficiency in chronic fatigue syndrome and multiple sclerosis. Arch Neurol 1993;50:301-4.

3 Beatty WW. Memory and frontal lobe dysfunction in multiple sclerosis. F Neurol Sci 1993;115:38-41.

14 DeLuca J, Gaudino EA, Diamond BJ, et al. Acquisition and seLuca J, Gaudino EA, Diamond BJ, et al. Acquisition and
storage deficits in multiple sclerosis. $\mathcal{F}$ Clin Exp Neuropsystorage deficits in muld
chol 1998;20:376-90.

15 Jonsson A, Korfitzen EM, Heltberg A, et al. Effects of neuropsychological treatment in patients with multiple sclerosis. Acta Neurol Scand 1993;88:394-400. 